\title{
LAMINATION SAMPLING TECHNIQUE FOR UNCONSOLIDATED SEDIMENTS
}

\author{
STEPHEN P. LEATHERMAN \\ Department of Geology, Boston University, Boston, Maine, U.S.A.
}

ALIAN T. WILIIAMS

The Polytechnic of Wales, Pontypridd, Mid Glamorgan U.K.

An adjustable shop wrench to which thin plexiglass squares were mounted as jaws was used to sample unconsolidated sediments. The jow opening could be set so as to match perfectly the thickness of the lamina to be sampled. Samples could be taken with little risk of contamination from adjacent strata and could be inspected before acceptance because of the transparency of the plexiglass slides.

\section{INTRODUCTION}

Sedimentologists have traditionally relied upon a pocket knife or nail file to pick grains from a desired lamination, or have excavated the lamination required for sampling purposes by stripping away overlying strata. These techniques are time-consuming if, as frequently is the case, a large sample is necessary for analysis and the unit to be sampled is thin. In addition, removing material from the side of a trench or pit wall may induce caving and consequent contamination. Material may be recovered from the upper or lower strata along with the desired sample without detection, even if caving is not a problem.

The sediment sampler was designed in order to alleviate these problems in sampling unconsolidated materials. Two $0.8 \mathrm{~mm}$ ( $1 / 32$ inch) thick plexiglass slides were glued to the jaws of a small-sized adjustable shop wrench (Fig. 1). It was the adjustability of the sampler that gave it the capability of obtaining samples from a wide range of lamina thicknesses. Each sample removed from a lamina could be visually inspected for impurities before placing into a sampling bag or vial.

\section{SAMPLING TECHNIQUE AND APPLICATION}

The wall of the trench or pit should be smoothed with a trowel. The distance between the plexiglass plates is then set so as to match the thickness of the lamination required and pressed into the deposit parallel to the plane of the strata. Once inserted, the wrench is tightened slightly so as to prevent loss of samples with removal. Even if the wall crumbles when pulling out the sampler, material can neither contaminate nor fall out of the jaws. After visul inspection of the sample from all sides for textural and mineralogical homogeneity, the sample is released by loosening the grip of the wrench. Then the sampler is washed in a small pail of water in order to remove the few remaining grains that adhere to either side of the plates and also to clean out the adjustable portion of the wrench.

The sampler was successfully used to obtain particles in the fine to medium sand size range from an overwash deposit (Leatherman and Williams 1977). It could be used for finer material, including cohesive material if not hardened by post-depositional changes. The upper range of effectiveness is probably limited to coarse quartz

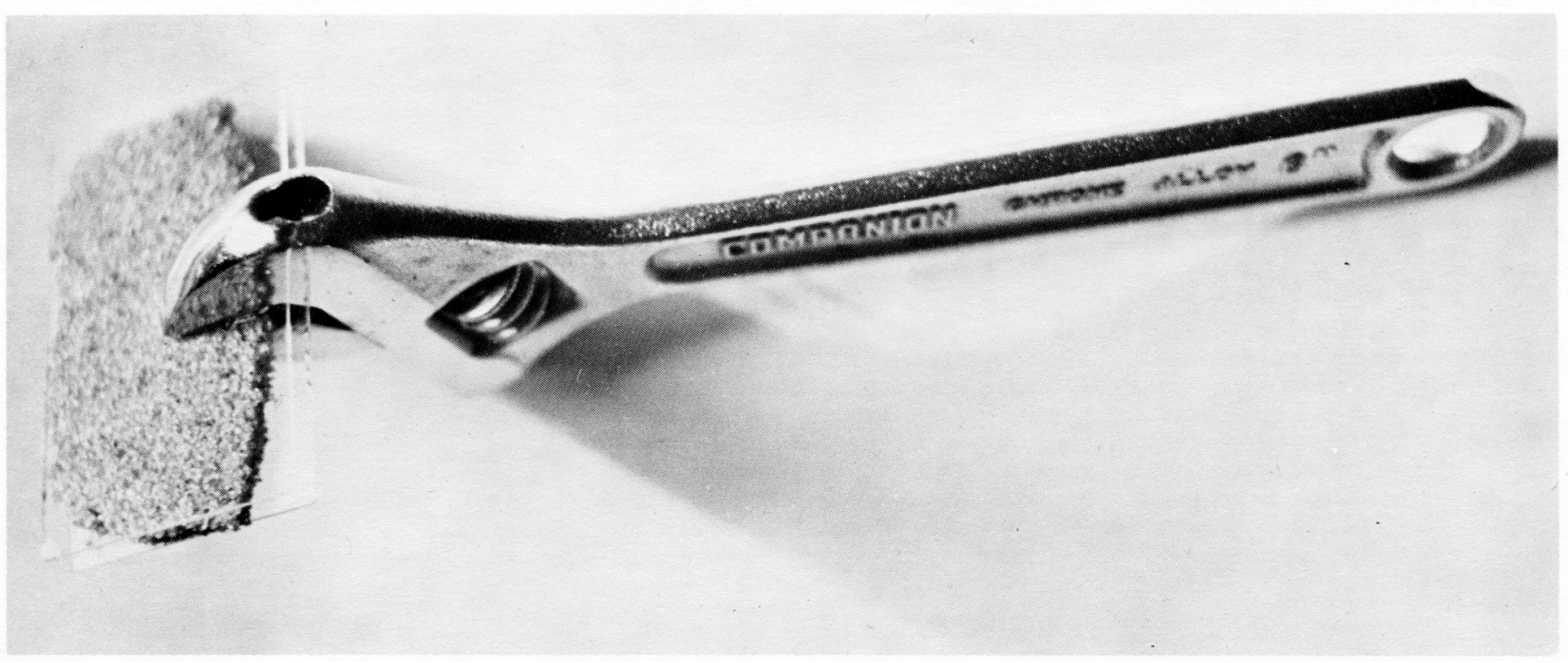

FIG. 1 Device for sampling laminations. 
since at this point the amount of pressure necessary to force the sampler into the strata may exceed the strength of the glue binding the plexiglass slides to the wrench opening. Also, a large sample in this size range can easily be obtained with a knife or spoon. Since the overwash sand was horizontally layered, there was no problem with laterial contamination. In the case of cross-bedding it would be necessary to limit the width of the plates as the sampler can be adjusted only in two dimensions. The penetration distance into the wall is usually not a factor since units are generally laterally homogeneous for more than several centimetres. The length of the unit may be a problem in a few limited cases, depending on the scale of the cross-beds.

\section{CONCLUSION}

This technique allowed for the attainment of a representative sample quickly and without contamination under field conditions. The cost is nominal and it can be produced with minimal effort. This adjustable wrench sampler should be useful for unconsolidated, cohesionless material in the sand size range or smaller. Silt and clay laminae could be sampled if diagenesis or dessication has not altered the original "soft" character of the material.

\section{REFERENCES}

LEATHERMAN, S.P. and WILLIAMS, A.T. 1977. Lateral grading in an overwash fan. Earth Surface Processes, 2. (4), pp. 333-342. 